\title{
UNA TASA DE CRECIMIENTO DEMOGRÁFICO NULO EN LOS PAÍSES EN VÍAS DE DESARROLLO AL AÑO 2000 , ¿SUEÑO O REALIDAD?*
}

\author{
JEAN BOURGEOIS-PICHAT \\ $\mathrm{y}$ \\ Si-AHMED TALEB \\ Instituto Nacional de Estudios Demográficos, Paris
}

El general William H. Draper fue nombrado por el presidente Nixon en 1969 representante de los Estados Unidos ante la Comisión de Población de las Naciones Unidas. En un almuerzo en su honor se pronunció a favor de una tasa de crecimiento nula de la población de los Estados Unidos (zero population growth rate) para fines del siglo, ejemplo que debería ser seguido por ctros países.

El texto de esta solicitud se publicó, en enero de 1970, en una Newsletter del Comité sobre la Crisis de la Población (Washington, D. C.). Aun cuando se refirió en primer lugar a los Estados Unidos, Draper también tenía en mente a los países en vías de desarrollo.

Es el caso de estos países el que aqui se examina; tomando como ejemplo la población de México, se estudian las consecuencias demográficas inesperacias de la evolución que se propone.

\section{Previsiones demográficas en la PRIMERa hipótesis}

Esta hipótesis, que denotamos con $H 1$ va de acuerdo con la evolución solicitada.

Hipótesis de base. Partiendo de los resultados del censo de población mexicano de 1960,1 hemos supuesto una disminución progresiva de la tasa de crecimiento de la población, de modo que este crecimiento sea nulo durante el período 1995-2000 y permanezca después a ese nivel. Las tasas anuales de crecimiento de la población que fueron adoptadas (por 1000 ) son las siguientes:

\begin{tabular}{|c|c|c|c|}
\hline $\begin{array}{l}1960-1965 \\
1965-1970 \\
1970-1975 \\
1975-1980\end{array}$ & $\begin{array}{l}32.8 \\
28.4 \\
24.0 \\
19.6\end{array}$ & $\begin{array}{l}1980-1985 \\
1985-1990 \\
1990-1995 \\
1995-2000\end{array}$ & $\begin{array}{r}15.4 \\
11.0 \\
6.6 \\
0.0\end{array}$ \\
\hline
\end{tabular}

* Este artículo apareció en Population, Núm. 5, septiembre-octubre de 1970 bajo el título. "Un Taux d'accroissement nul pour les pays en voie de développenent en l'an 2000. Rêve ou réalité?" La traducción es de A. García Rocha.

1 Se levantó un censo en enero de 1970 , pero en el momento de hacer estos cálcuios sólo se habían publicado resultados globales. 
En cuanto a la mortalidad, hemos supuesto un aumento sostenido de la esperanza de vida al nacer. Hasta 1990 hemos utilizado las tablas de vida adoptadas por las autoridades mexicanas, para las proyecciones de la población de su país. ${ }^{2}$ Después de 1990, el descenso de la mortalidad ha sido calculado por medio de las tablas de supervivencia tipo de las Naciones Unidas, tomando para el período 1990 1995, de la tabla tipo del sexo femenino el nivel 105 y para el periodo 1995-2000 el nivel 110. La esperanza de vida al nacimiento, correspondiente a estas hipótesis, expresada en años, es como sigue:

\begin{tabular}{llll}
\hline $1960-1965$ & 60.3 & $1980-1985$ & 69.5 \\
$1965-1970$ & 62.2 & $1985-1990$ & 71.4 \\
$1970-1975$ & 65.3 & $1990-1995$ & 73.2 \\
$1975-1980$ & 67.6 & $1990-2000$ & 74.3 \\
\hline
\end{tabular}

Después del año 2000 las tasas de mortalidad por edad permanecen constantes.

Mediante estas hipótesis acerca de la tasa anual de crecimiento de la población y acerca de la mortalidad, es fácil establecer la proyección de la población mexicana. Los cálculos fueron elaborados hasta el año 2055 .

Algunas observaciones generales. Antes de presentar los resultados de los cálculos, es útil hacer algunas observaciones acerca de las previsiones de población. Para hacer tal proyección, es preciso formular una hipótesis acerca de algunas características de la población. Estas características son los datos de base de la previsión y es usual prever que éstas seguirán una evolución regular y sin cambios abruptos. Las otras características resultan de la proyección misma; sus rasgos futuros no son tan regulares y, por lo general, muestran oscilaciones desde un principio.

Por ejemplo, las hipótesis se refieren a la tendencia de las tasas de mortalidad por edad, las tasas de fecundidad femenina y los resultados sobre la población total, su composición por edad, la tasa bruta de natalidad, etc. La evolución de las características de base se suponen regulares, pero las características resultantes oscilan. Estas ascilaciones se amortiguan con el tiempo y finalmente alcanzan un estado en el que todas las características de la población evolucionan de manera regular. Esta situación es denominada estable. Cuando la población total se mantiene constante, la situación se llama estacionaria.

De entre las diversas características de la población, dos de ellas no pueden oscilar de manera importante: la mortalidad y la fecundidad. Ésta es la razón por la cual ambas características figuran por lo general entre los datos base de la proyección.

El nivel de la fecundidad. He aquí el índice sintético de fecundidad femenina ${ }^{3}$ (por 1000) determinado por las dos hipótesis de Draper:

2 Raúl Benítez Zenteno y Gustavo Cabrera Acevedo, Proyecciones de la población de México 1960-1980, Banco de México, 1966.

3 Número de nacimientos femeninos en un año, en relación con la población femenina de 15 a 49 años de edad. 


\begin{tabular}{lrllll}
\hline $1960-1965$ & 93.8 & $1995-2000$ & 8.6 & $2030-2035$ & 46.2 \\
$1965-1970$ & 9.9 & $2000-2005$ & 9.9 & $2035-2040$ & 55.5 \\
$1970-1975$ & 5.3 & $2005-2010$ & 11.9 & $2040-2045$ & 59.8 \\
$1975-1980$ & 51.4 & $2010-2015$ & 15.2 & $2045-2050$ & 56.5 \\
$1980-1985$ & 39.7 & $2015-2020$ & 20.3 & $2050-2055$ & 48.8 \\
$1985-1990$ & 29.0 & $2020-2025$ & 27.3 & Bstado estacio & \\
$1990-1995$ & 19.7 & $2025-2030$ & 36.1 & nario & 30.0 \\
\hline
\end{tabular}

La gráfica 1 traduce esta evolución en la curva $H 1^{4}$

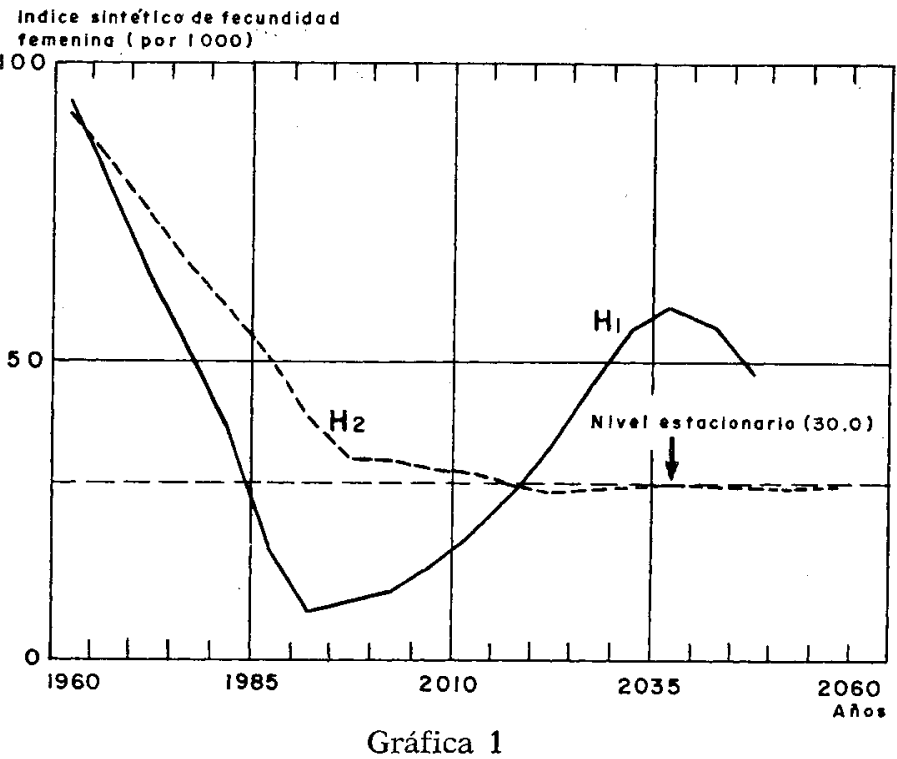

México: Proyección de la poBLACIÓN FEMENINA. ÍNDICE SINTÉTICO DE FECUNDIDAd FEMENINA (NÚMERO ANUAL DE NACIMIENTOS FEMENINOS CORRESPONDIENTES A LA POBLACIÓN FEMENINA DE 15 A 49 AÑOS DE EDAD)

La tasa de fecundidad oscila con gran amplitud. El punto más bajo, en 1995-2000, corresponde a 0.6 hijos por mujer, y el más alto, en 2040-2045 a 3.8. Estas variaciones de la fecundidad son verdaderamente imposibles de concebir. Imaginar que la fecundidad de las mujeres mexicanas se multiplique 6 veces de 1995-2000 a 2040-2045 no resulta realista. No está claro qué tipo de decisiones podrían tomar las autoridades mexicanas para lograr tal resultado. Por otra parte, después de 2040-2045, habría que tomar otras decisiones en sentido inverso, con el fin de reducir la fecundidad.

En vista de que, según las hipótesis, la población total debe permanecer constante después del año 2000 , se alcanzará un estado estacionario. A este estado estacionario corresponde una fecundidad total de 30 por 1000 , lo cual significa que antes de alcanzar este nivel, la

4 En esta gráfica se muestra otra curva llamada $H 2$. En muchas de las otras gráficas sucederá lo mismo pero por el momento consideraremos solamente $H 1$; las curvas $H 2$ se examinarán después. 
fecundidad total deberá oscilar alrededor de él. La gráfica 1 muestra sólo dos oscilaciones; pero si se continuaran más allá del año 2055, observaríamos otras oscilaciones, que se amortiguarían muy lentamente hacia el nivel estacionario final.

- En resumen, se puede decir que suponer una tasa de crecimiento nuta en el año 2000 y más allá de éste, crea en el caso de la fecundidad oscitaciones contrarias a la naturaleza misma de este fenómeno. Esta comprobación podría ser suficiente para considerar la hipótesis como irreal y abandonarla. Sin embargo, es interesante examinar los efectos de esta hipótesis sobre otras características demográficas.

La población total. Esta evidentemente no sufre oscilaciones, dado que uno de los datos de la proyección es mantenerla constante después del año 2000. Este nivel constante es 34537946 para el caso de la población femenina. La total sería casi el doble de esa cantidad.

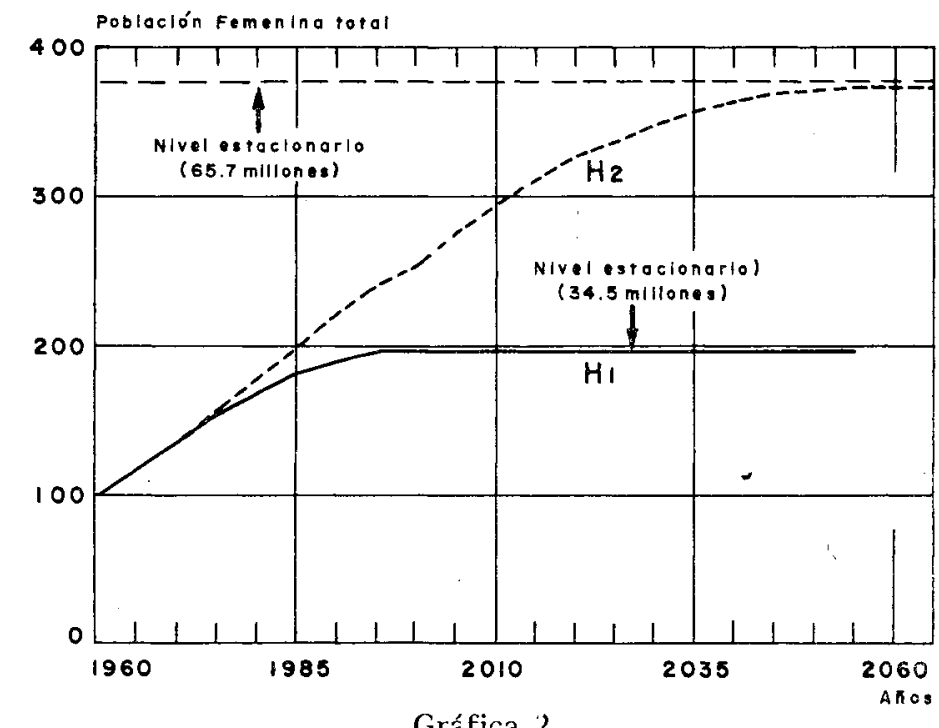

México: Proyección de la población femenina. Población femenina total (BASE 100 EN 1960: 17.5 MILLONES)

La gráfica 2 muestra que la población femenina se duplica de 1960 a 2000 (curva H1). La población inicial de 17.5 millones en 1960 es tomada como base 100 .

Pero si la población total no oscila, algunas fracciones de ésta su-

\begin{tabular}{|c|c|c|c|c|c|}
\hline $\begin{array}{l}1960 \\
1965 \\
1970 \\
1975 \\
1980 \\
1985 \\
1990\end{array}$ & $\begin{array}{l}4.7 \\
5.3 \\
6.3 \\
7.2 \\
7.2 \\
6.9 \\
6.4\end{array}$ & $\begin{array}{l}1995 \\
2000 \\
2005 \\
2010 \\
2015 \\
2020 \\
2025\end{array}$ & $\begin{array}{l}5.6 \\
4.5 \\
2.9 \\
2.0 \\
2.3 \\
2.8 \\
3.2\end{array}$ & $\begin{array}{l}2030 \\
2035 \\
2040 \\
2045 \\
2050 \\
2055 \\
\text { Es tado } \\
\text { entecionario }\end{array}$ & $\begin{array}{l}3.7 \\
4.3 \\
4.9 \\
5.4 \\
5.9 \\
6.2 \\
4.6\end{array}$ \\
\hline
\end{tabular}


BOURGEOIS-PICHAT/TALEB : CRECIMIENTO DEMOGRAFICO NULO 81

frirán, por el contrario fuertes oscilaciones, como veremos en seguida.

Población en edad escolar. La variación de la población femenina de 5 a 14 años fluctúa como se muestra en el cuadro que precede (en millones). Las cifras son reproducidas en la curva $H 1$ de la gráfia 3 .

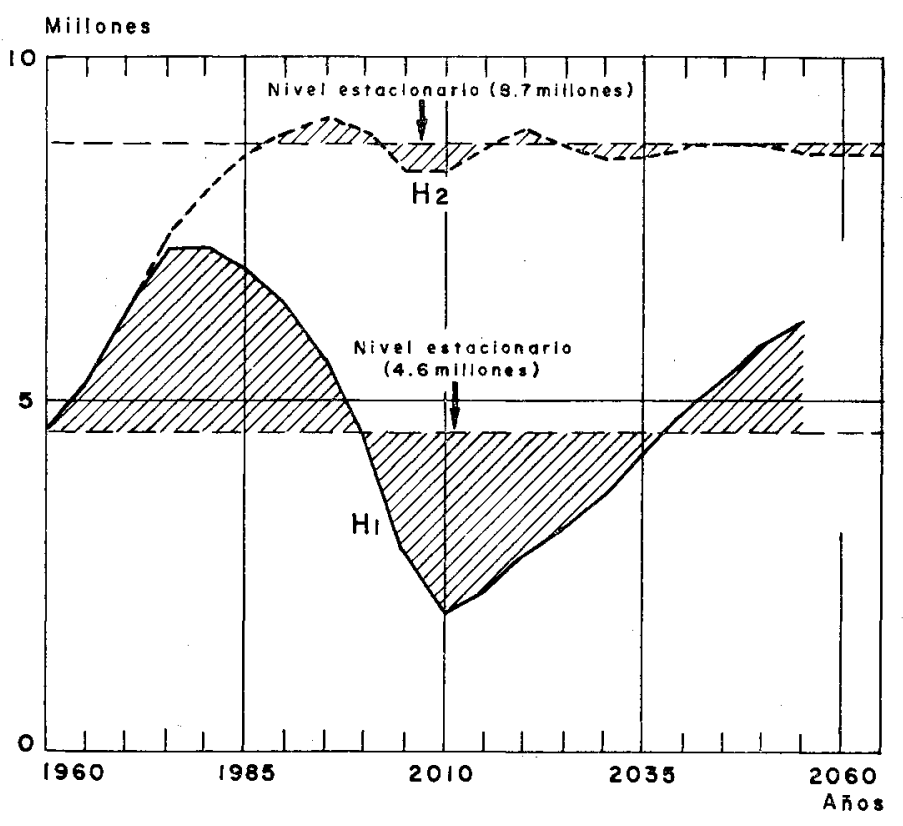

Gráfica 3

México: Proyećción de la población femenina. Población en edad ESCOLAR: 5-14 AÑOS.

La población en edad escolar oscila entre 2 y 7.2 millones, alrededor de un nivel estacionario de 4.6 millones, y esto indica claramente las dificultades que resultarían de tales variaciones. En el período 20102015, menos de un tercio del equipo necesario 30 años antes (en 1980-1985) bastaría a la población escolar y 50 años después sería necesario volver a una situación muy semejante a la de 1980-1985. Por otra parte, tales oscilaciones continuarían durante mucho tiempo. En tal situación, la formación de maestros sería imposible.

Población en edad activa. La evolución de la población femenina de 20 a 64 años (en millones), es como sigue:

\begin{tabular}{lrllll}
\hline 1960 & 7.2 & 1995 & 21.7 & 2030 & 20.1 \\
1965 & 8.7 & 2000 & 23.7 & 2035 & 18.0 \\
1970 & 10.2 & 2005 & 25.2 & 2040 & 16.3 \\
1975 & 12.0 & 2010 & 25.9 & 2045 & 15.1 \\
1980 & 13.9 & 2015 & 25.7 & 2050 & 14.5 \\
1985 & 16.6 & 2020 & 24.0 & 2055 & 14.7 \\
1990 & 19.2 & 2025 & 22.3 & Estado & 19.5 \\
\hline
\end{tabular}




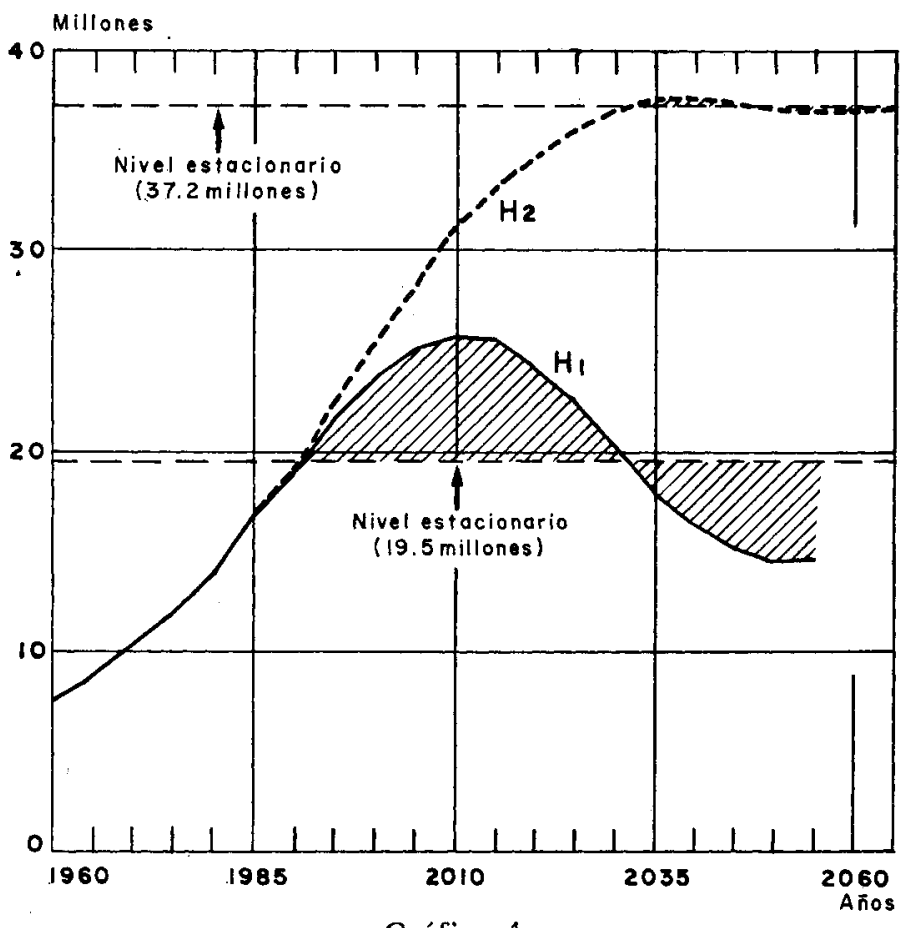

Gráfica 4

México: Proyección de la población femenina. Población en edad ACTIVA : $20-64$ AÑoS

Hasta 1980, esta población ya había nacido, si el punto de partida del cálculo es 1960. Es sólo después de 2025 que estará totalmente compuesta por personas nacidas con posterioridad a esta época (en 2025 las personas nacidas en 1960 tendrán 65 años). Es por esto que habría sido necesario prolongar la proyección más allá del año 2055 con objeto de tener una idea clara de las amplitudes de estas oscilaciones. Pero la gráfica 4 (curva $H 1$ ) muestra ya una oscilación del $30 \%$ por encima y por debajo del nivel estacionario.

Todo plan de desarrollo económico se desplomaría con tal evolución de la población activa.

Población de edades superiores. Las oscilaciones de la población de edades superiores ( 65 y más años) planteará asimismo grandes dificultades. He aquí, en millones, los resultados correspondientes a esta población particular.

\begin{tabular}{llllll}
\hline 1960 & 0.6 & 1995 & 1.9 & 2030 & 6.9 \\
1965 & 0.7 & 2000 & 2.4 & 2035 & 7.9 \\
1970 & 0.8 & 2005 & 2.9 & 2040 & 8.5 \\
1975 & 1.0 & 2010 & 3.4 & 2045 & 8.6 \\
1980 & 1.2 & 2015 & 4.1 & 2050 & 8.3 \\
1985 & 1.4 & 2020 & 5.0 & 2055 & 7.6 \\
1990 & 1.7 & 2025 & 5.7 & Iivel & \\
& & & & ostecionsirio & 5.9 \\
\hline
\end{tabular}




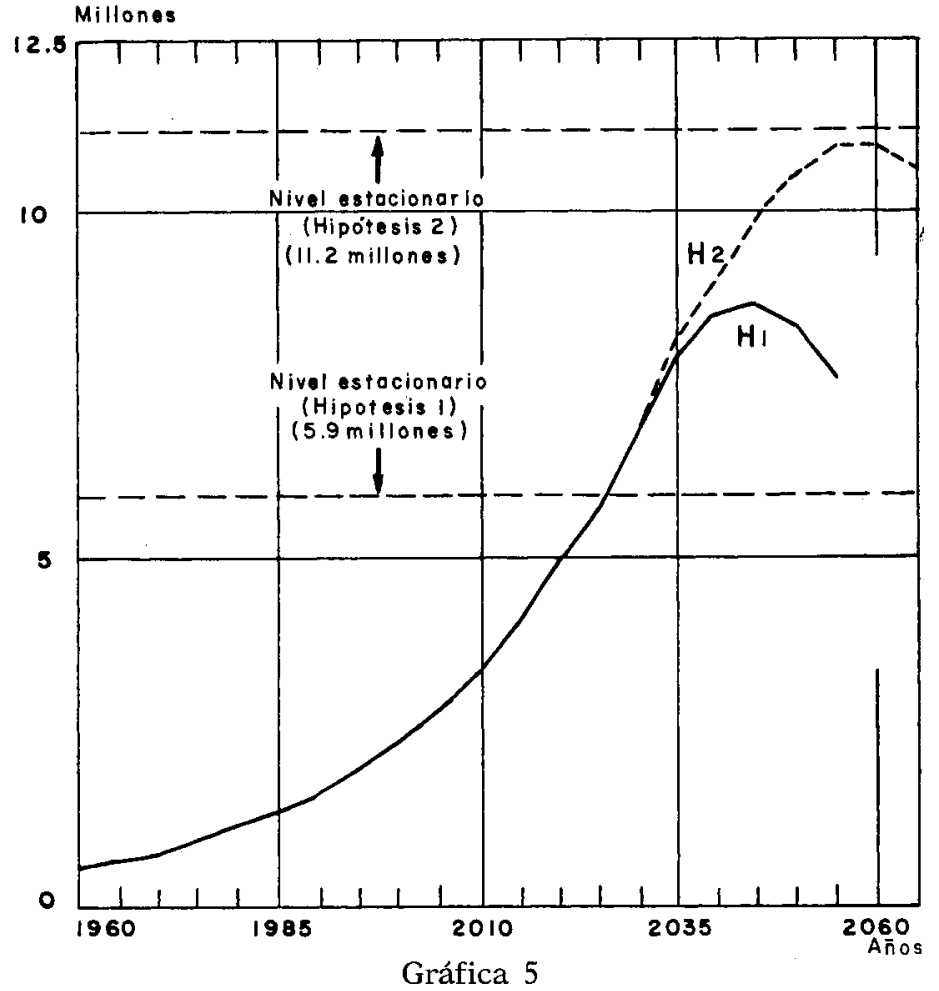

México: Proyección de la población femenina. Poblactón en edades SUPERIORES: 65 AÑNOS Y MÁS.

La figura 5 representa estos datos. Limitando los cálculos al año 2055, sólo podemos observar el principio de la primera oscilación

\begin{tabular}{lllcl}
\hline Años & $0-19$ & $20-64$ & 65 y mśs & Total \\
\hline 1960 & 53.7 & 42.8 & 3.5 & 100.0 \\
1965 & 54.3 & 42.2 & 3.5 & 100.0 \\
1970 & 53.5 & 43.0 & 3.5 & 100.0 \\
1975 & 59.1 & 45.1 & 3.8 & 100.0 \\
1980 & 48.3 & 47.6 & 4.1 & 100.0 \\
1985 & 43.1 & 52.5 & 4.4 & 100.0 \\
1990 & 37.5 & 57.5 & 5.0 & 100.0 \\
1995 & 31.7 & 62.7 & 5.6 & 100.0 \\
2000 & 24.5 & 68.6 & 6.9 & 100.0 \\
2005 & 18.8 & 72.9 & 8.3 & 100.0 \\
2010 & 15.0 & 75.1 & 9.9 & 100.0 \\
2015 & 13.7 & 74.5 & 11.8 & 100.0 \\
2020 & 16.1 & 69.6 & 14.3 & 100.0 \\
2025 & 18.8 & 64.7 & 16.5 & 100.0 \\
2030 & 21.8 & 58.2 & 20.0 & 100.0 \\
2035 & 24.9 & 53.2 & 22.8 & 100.0 \\
2040 & 28.2 & 47.3 & 24.5 & 100.0 \\
2045 & 31.2 & 43.9 & 24.9 & 100.0 \\
2050 & 33.7 & 42.1 & 24.2 & 100.0 \\
2055 & 35.4 & 42.5 & 22.1 & 100.0 \\
Nive1 estacionario & 26.4 & 56.6 & 17.0 & 100.0 \\
\hline
\end{tabular}


(cuyo máximo se alcanza en 2045). Pero la comparación con el nivel estacionario muestra que son posibles las oscilaciones de $50 \%$ por encima y por debajo de este nivel. Ningún sistema de jubilaciones podria funcionar con tales oscilaciones.

La composición por edades. Es igualmente interesante ver cómo se comportan en la proyección los diversos grupos de edad. El cuadro de la p. 83 muestra las variaciones de tres grandes grupos de edad, 0-19 años, 20-64 años y 65 y más años (véase también la gráfica 6).

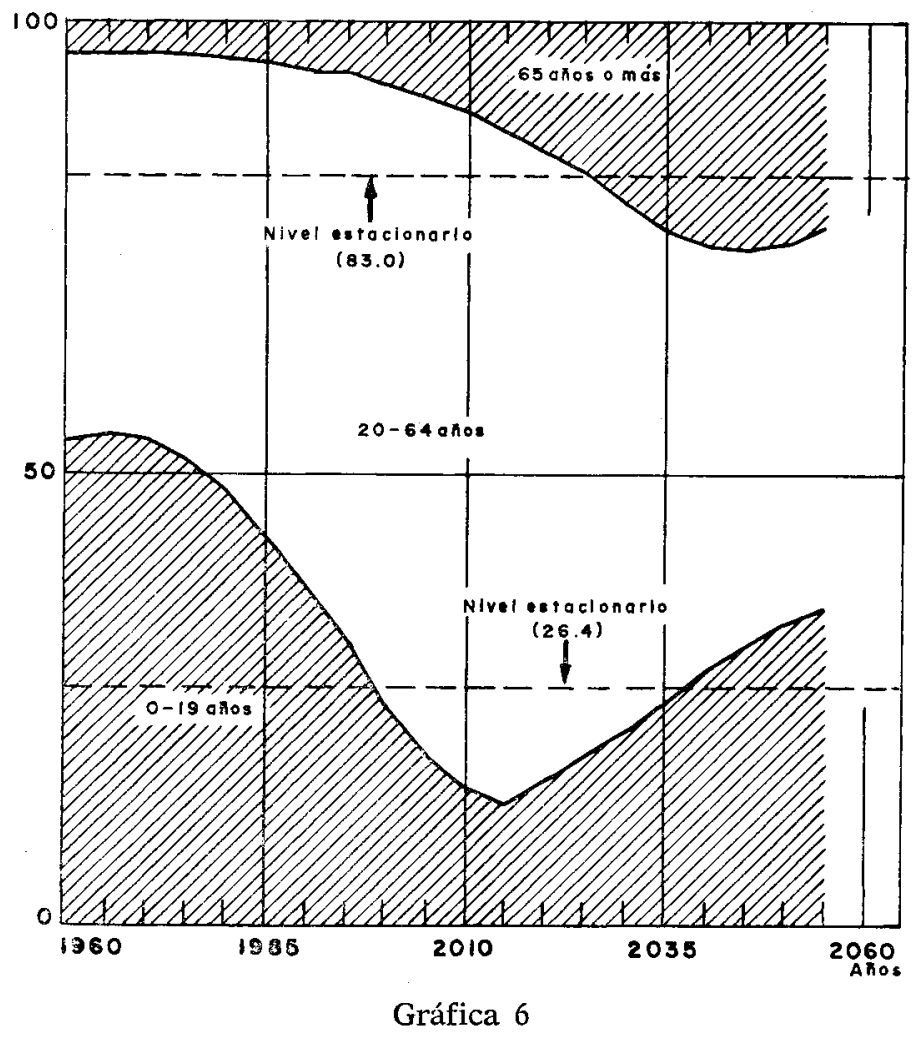

México: Proyección de la población FEMENina. CoMposición POR EDADES DE LA POBLACIÓN (HIPÓTESIS H1)

Las oscilaciones alrededor del nivel estacionario aparecen claramente. Como en los otros casos, estas oscilaciones se desvanecen poco a poco en el tiempo, pero el amortiguamiento será lento y la composición por edades continuará oscilando mucho tiempo después de 2055 (durante varias centenas de años).

Entradas a la población economucamente activa y salidas por retino $y$ jubilación. Un quinto de la población de 15-19 años, dividido por la población en edad activa, da una estimación de la tasa anual de 
entrada a la población económicamente activa. La misma operación, con un quinto de la población de 65-69 años, da una estimación de la tasa anual de salida por jubilación o retiro. Veamos los resultados expresados en miles:

\begin{tabular}{ccc}
\hline Años & Entradas & Salidas por retiro \\
\hline $1960-1965$ & 48.2 & 5.6 \\
$1965-1970$ & 48.7 & 7.6 \\
$1970-1975$ & 50.7 & 6.3 \\
$1975-1990$ & 45.2 & 6.9 \\
$1980-1985$ & 50.9 & 6.7 \\
$1985-1990$ & 43.8 & 6.1 \\
$1990-1995$ & 37.0 & 7.2 \\
$1995-2000$ & 30.9 & 7.0 \\
$2000-2005$ & 25.8 & 8.1 \\
$2005-2010$ & 20.4 & 9.1 \\
$2010-2015$ & 14.9 & 10.4 \\
$2015-2020$ & 7.1 & 12.5 \\
$2020-2025$ & 8.9 & 16.6 \\
$2025-2030$ & 11.3 & 19.1 \\
$2030-2035$ & 14.8 & 27.9 \\
$2035-2040$ & 15.1 & 32.0 \\
$2040-2045$ & 24.5 & 32.0 \\
$2045-2050$ & 30.2 & 35.0 \\
$2050-2055$ & 35.4 & 33.3 \\
$2055-2060$ & 38.8 & 27.8 \\
Nivel estacionario & 23.2 & 18.4 \\
\hline
\end{tabular}

Las oscilaciones de las gráficas 7 y 8 muestran cómo se desorganizaría la población económicamente activa.

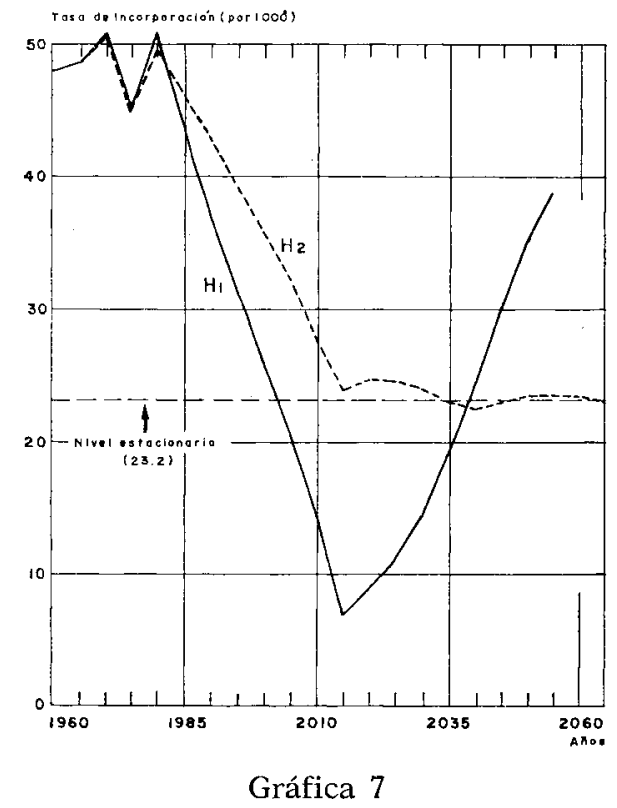

México: Proyección de la población femenina. Estrmación de las tasas DE ENTRADA A LA POBLACTÓN ACTIVA. 


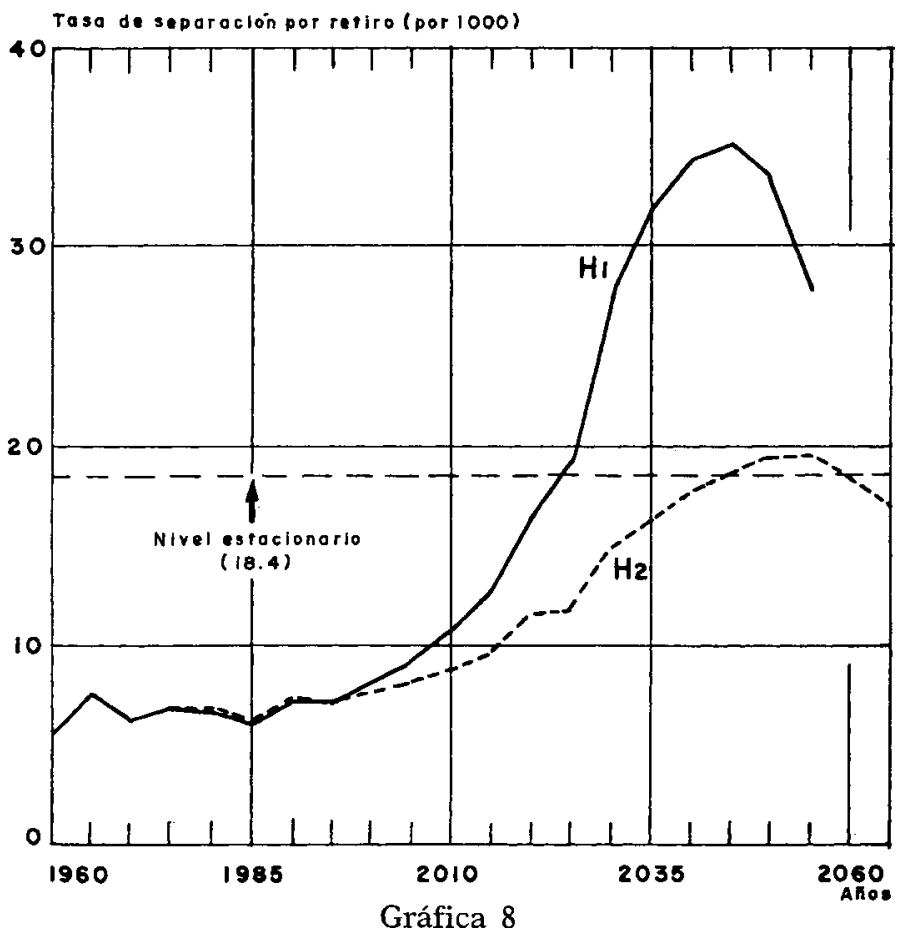

MÉXICo: Proyección de la población femenina. Estimación DE TASAS DE SALIDA POR RETIRO

Las tasas brutas de mortalidad y de natalidad. La aplicación de tasas constantes de mortalidad por edades a una población cuya composición por edades cambia, da una oscilación de la tasa de mortalidad. Después del año 2000, la tasa bruta de natalidad es, por hipótesis, igual a la tasa bruta de mortalidad. Encontraremos pues las mismas oscilaciones en las dos tasas. He aquí los resultados (por $1000)$ :

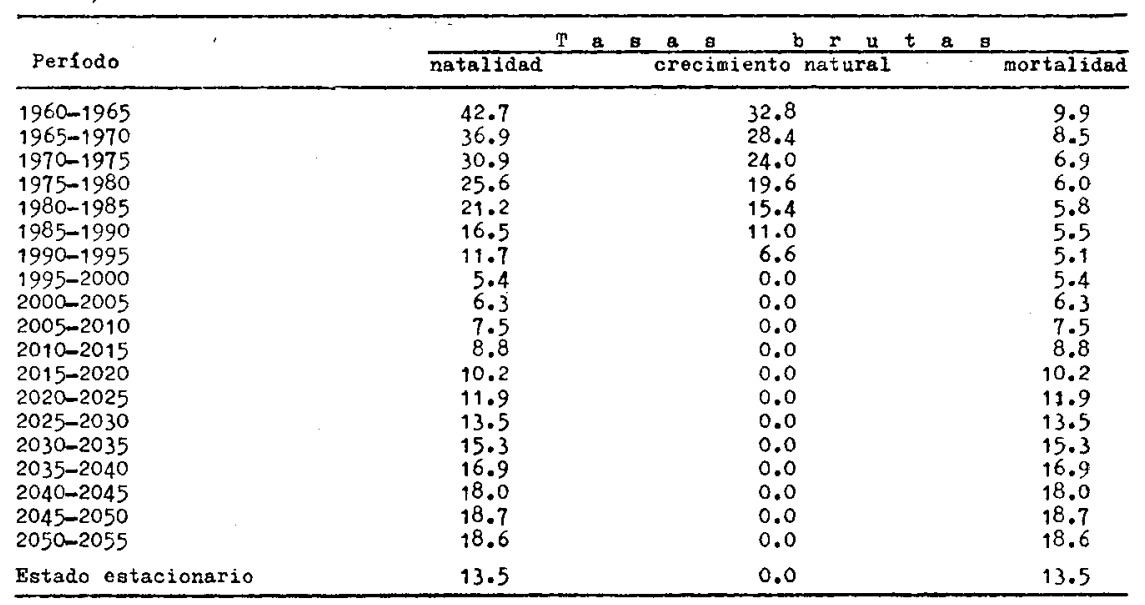




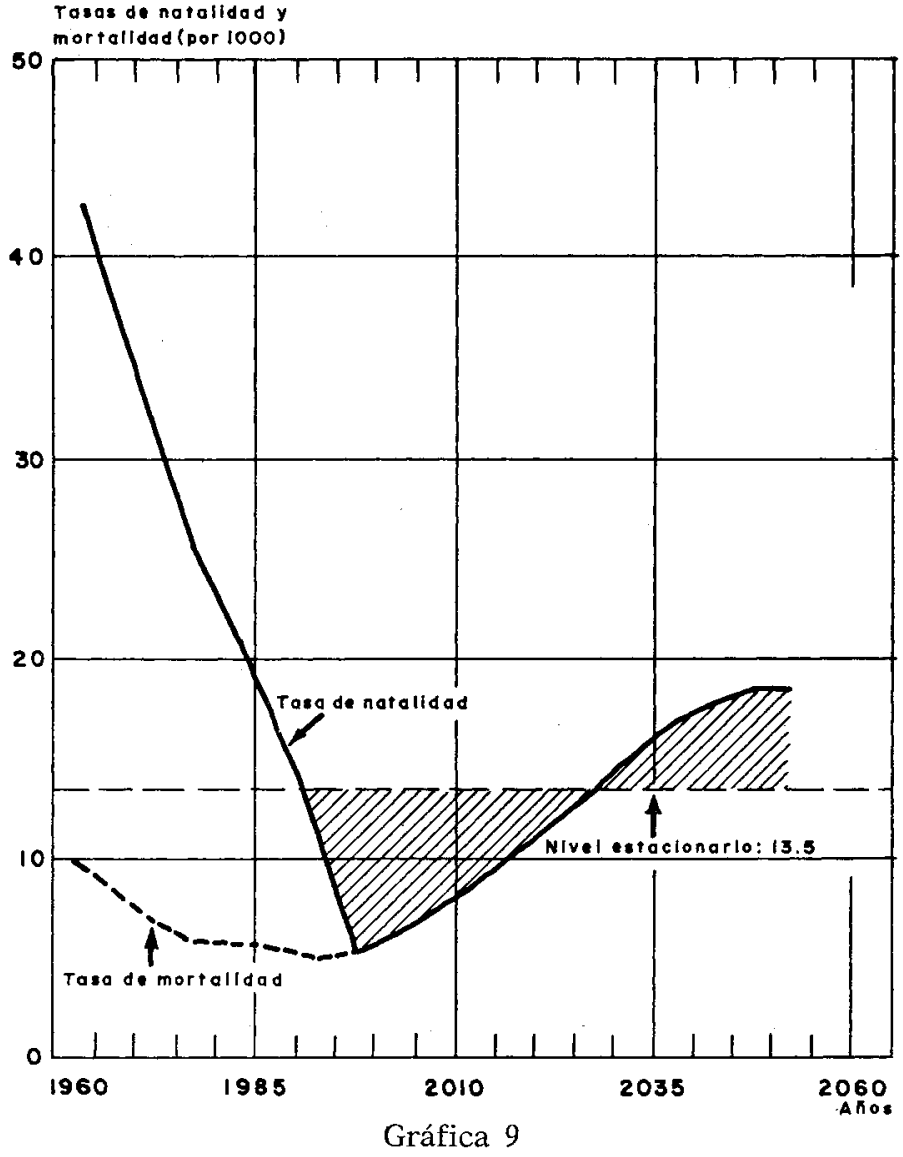

MÉxico: Proyección de la POBLación FEMENINa. Tasa bRUta de Natalidad Y TASA BRUTA DE MORTALIDAD (HPÓTESIS H1)

Estos resultados muestran además en qué medida la tasa bruta de mortalidad será un índice poco adecuado de la importancia de la mortalidad. Más allá de 1995-2000, el nivel de la mortalidad es constante (la esperanza de vida es igual a 75.2 años). A pesar del mantenimiento del nivel constante y en razón de los cambios en la composición por edades, la tasa bruta de mortalidad se multiplica por 3.5 de $1995-2000$ a 2045-2050.

En todas estas proyecciones, la ley de disminución de la tasa de crecimiento de la población hasta llegar a cero en el año 2000 tiene poca influencia sobre la amplitud y la duración de las oscilaciones. Finalmente, si los cálculos se hicieran a partir de 1970 en Iugar de 1960, las oscilaciones serían todavía mayores.

El análisis se detiene aquí. El lector está seguramente convencido ahora de que no es realista asignar a un país en vías de desarrollo un 
objetivo de crecimiento demográfico nulo para el año 2000 y que continúe luego a ese nivel.

En primer lugar, la naturaleza de los fenómenos demográficos se opone a tal objetivo, el cual no puede ser alcanzado sino a través de medidas artificiales que inciten a las parejas a modificar su fecundidad de modo tal que la familia completa promedio pase de 0.6 a 3.8 hijos y a la inversa, en un período de 50 años. Es difícil imaginar cuales podrían ser estas medidas.

Además, aun cuando estas medidas pudieran ser aplicadas, las diversas características de la población oscilarían de tal modo que toda la vida económica y social del país se vería trastornada por completo.

\section{UN OBJETIVO MÁS RAZONABLE}

Se plantea entonces una pregunta: en el mismo espíritu de la formulada por el general Draper ¿qué objetivo razonable puede ofrecerse al mundo, entre nuestros días y el año 2000? La falla principal de la previsión $H 1$ proviene del hecho de que la fecundidad no figura entre los datos base del cálculo. Para evitar esta falla, basta pues incluir la cundidad entre los datos base del pronóstico y admitir, respecto a ella, una tendencia tan regular y continua como sea posible. Al ser el objetivo final una población estacionaria ( $\mathrm{y}$ por lo tanto constante), se puede admitir que la fecundidad disminuya en forma regular desde hoy hasta el año 2000, de modo que en este último año la reproducción de la población (la renovación de las generaciones) sea apenas alcanzada y que a partir de ese momento la fecundidad permanezca invariable.

En términos demográficos, lo anterior significa que la tasa neta de reproducción sea igual a 1 a partir del año 2000. La hipótesis de reducción de la mortalidad puede conservarse sin inconvenientes.

El hecho de que la tasa neta de reproducción sea igual a 1 el año 2000 y permanezca a este nivel no significa que la población dejará de crecer después de este año. Dado que la composición por edades es favorable la tasa bruta de natalidad será todavía, durante algún tiempo, superior a la tasa de mortalidad, de modo que la población continuará creciendo. Con el tiempo, la composición por edades se hará cada vez menos favorable, el crecimiento de la población será cada vez más débil y finalmente se detendrá.

La fecundidad que corresponde a tal evolución conducirá, el año 2000, a la existencia de una familia completa con un número de hijos un poco superior a 2. Este número corresponde al deseo de las parejas en los paises industriales, lo cual es buena razón para escoger este nivel como aquel que se estabilizará después del año 2000 .

Con estas dos hipótesis sobre la mortalidad y la fecundidad, es posible establecer la proyección de la población. Los resultados se derivan de las cifras expuestas antes, mediante las curvas señaladas con H2. Las características demográficas observadas oscilarán como en la proyección anterior, pero estas oscilaciones serán mucho menos amplias que antes y, por encima de todo, las características de base, fecundidad y mortalidad, no oscilarán. 
Los resultados. Comentemos rápidamente los resultados de esta segunda proyección refiriéndonos a la curva $H 2$ de las gráficas precedentes.

En la gráfica 1, el nivel de fecundidad se estabiliza ${ }^{5}$ después del año 2000. Esto no debe extrañar, ya que esta condición figura entre los datos base del pronóstico.

En la gráfica 2, el nivel estacionario de la proyección $H 2$ es 65732000 habitantes de sexo femenino, cercano a lo alcanzado el año 2050. La población se multiplica entonces por 4, en lugar de 2 según la previsión $H 1$.

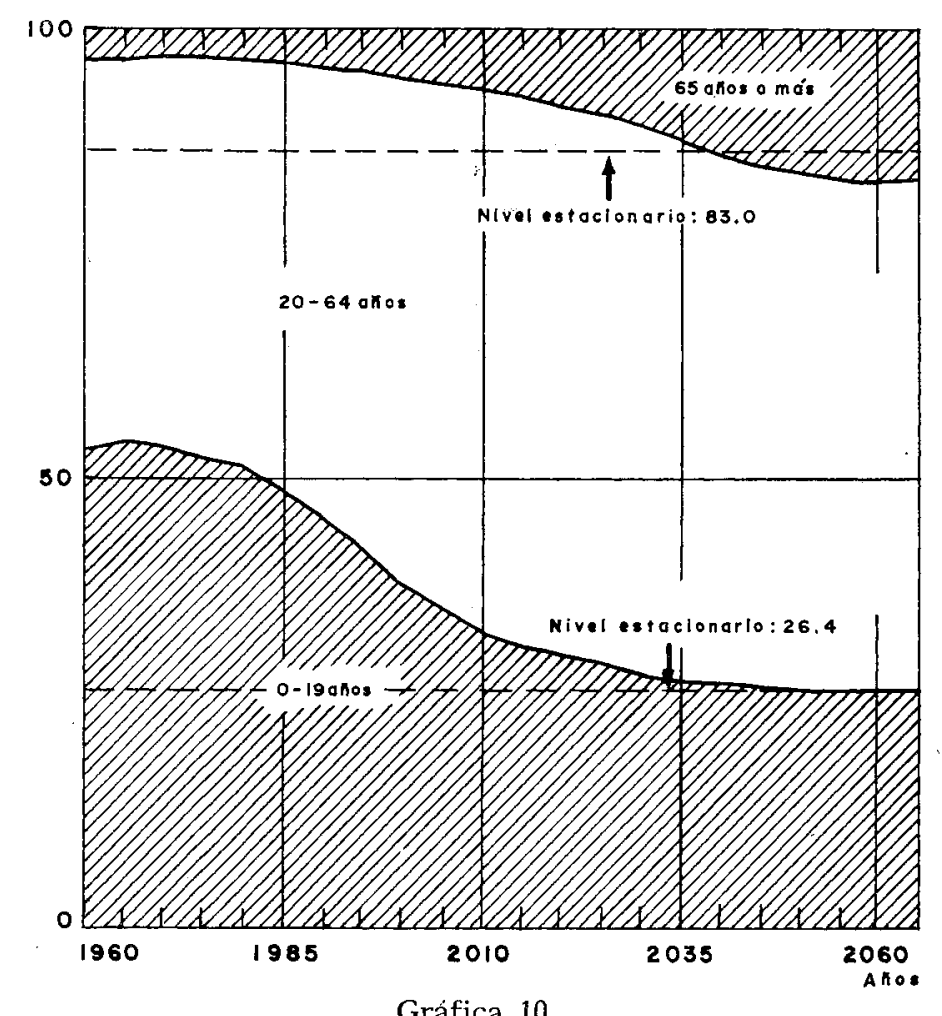

MÉXico: Proyección de la poblactón fEMEnina. Composición pOR edades DE LA POBLACIÓN (HIPÓTESIS H2)

En la gráfica 3, se observa, respecto de la curva $H 2$, que las oscilaciones se producen en la población en edad escolar, pero su ampli-

5 En la hipótesis de fecundidad de la previsión H2, las tasas de fecundidad por edad permanecen constantes después del año 2000. En la gráfica 1, la fecundidad se mide por medin de la fecundidad femenina total, que no es otra que la fecundidad femenina del grupo de edad 15-49 años. La constancia de las tasas de fecundidad 15-19, 20-24... 45-49 años, no implica la constancia de las tasas de fecundidad 15-49 años. Esto explica por qué, sobre la gráfica 1, la curva $H 2$ no es exactamente una línea horizontal después del año 2000, y pueden observarse todavia oscilaciones pequeñas. 
tud es relativamente débil y desaparecen muy rápidamente; el año 2050 son insignificantes y el estado estacionario se ha alcanzado.

Igual observación se hace para la gráfica 4 (población en edad activa). Llama especialmente la atención el contraste entre las curvas $H 2$ y $H 1$.

En la gráfica 5, relativa a la población en edades superiores, la diferencia entre $H 1$ y $H 2$ no es tan marcada; esto se debe a que no se continuó con el cálculo más allá del año 2055.

En cuanto a la composición por edades, se diseñó una nueva gráfica (10).

La comparación de las gráficas 6 y 10 muestra con claridad que en H2 se alcanza rápidamente un estado estacionario y de un modo regular y continuo. La misma evolución se observa en las entradas a la población activa y en las salidas por retiro o jubilación (gráficas 7 y 8 ).

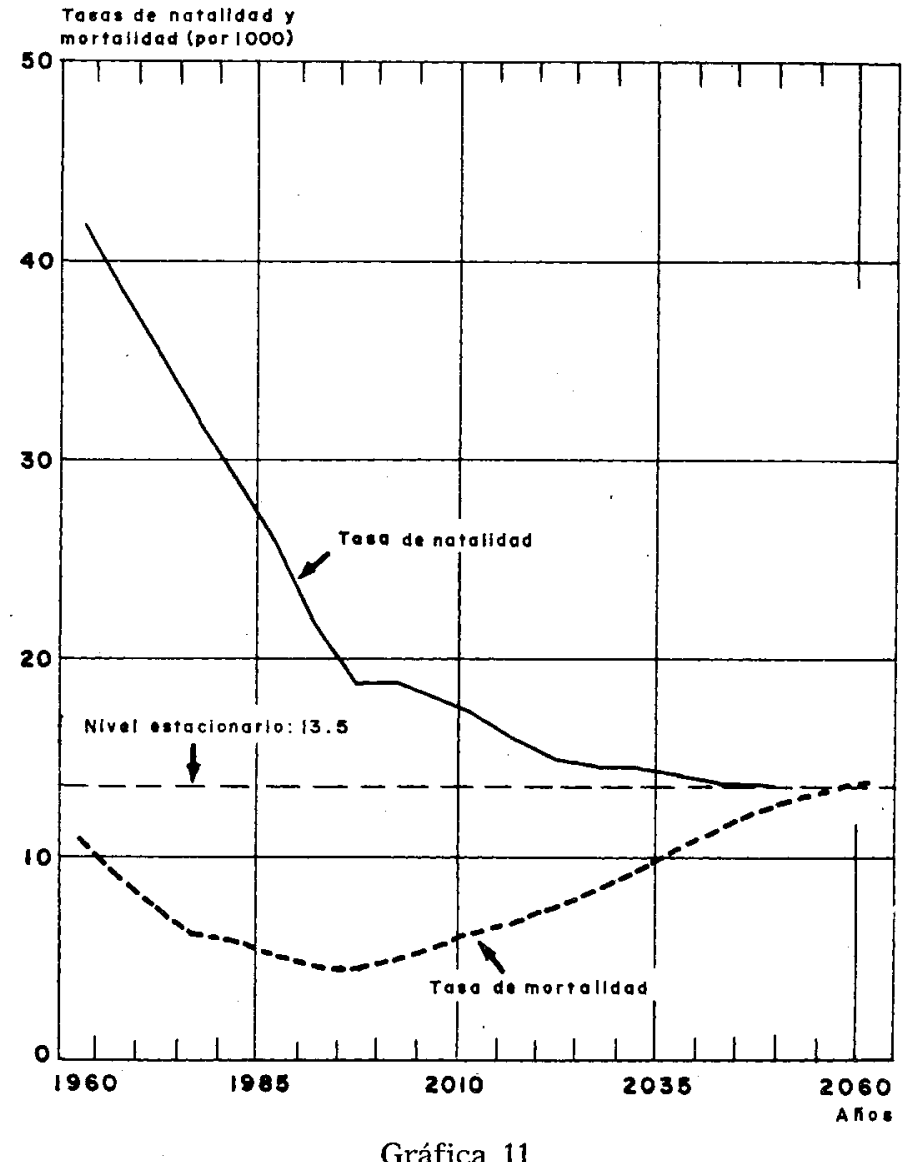

México: Proyección de la poblactón femenina. Tasa bruta de Natalidad Y TASA BRUTA DE MORTALIDAD (HIPÓTESIS H2) 


\section{BOURGEOIS-PICHAT/TALEB : CRECIMIENTO DEMOGRAFICO NULO 91}

De la comparación de los resultados $H 1$ y $H 2$ se desprende una conclusión muy clara: mientras que en $H 1$ la vida económica y social se vería gravemente perturbada, nada realmente serio desde este mismo punto de vista ocurre en la proyección $H 2$.

Por último, la gráfica 11 muestra la evolución de las tasas brutas de natalidad y mortalidad; esta evolución debe compararse con la de la gráfica 9.

Posibilidades de realización de la proyección $H 2$. Es interesante comparar la disminución supuesta de las tasas de natalidad de 1960 a 2000 con la disminución efectiva que ha aparecido recientemente en algunos países en vías de desarrollo. He aquí las tasas brutas de natalidad (por 1000 ) observadas en Singapur, Formosa y Puerto Rico:

\begin{tabular}{|c|c|c|c|c|c|c|}
\hline Año: & Singapur & Formona & Puerto Rioo & Perlodo & $\frac{M}{\bar{I} 1}$ & $\frac{0}{112}$ \\
\hline $\begin{array}{l}1953 \\
1958 \\
1963 \\
1968 \\
1968\end{array}$ & $\begin{array}{l}45.8 \\
42.0 \\
34.7 \\
24.7 \\
22.9\end{array}$ & $\begin{array}{l}45.2 \\
41.7 \\
36.3 \\
29.3 \\
25.6\end{array}$ & $\begin{array}{l}35.3 \\
32.2 \\
30.7 \\
25.1 \\
24.5\end{array}$ & $\begin{array}{l}1960-1965 \\
1965-1970 \\
1970-1975 \\
1975-1980\end{array}$ & $\begin{array}{l}42.7 \\
36.9 \\
30.9 \\
25.6\end{array}$ & $\begin{array}{l}41.9 \\
38.8 \\
35.4 \\
32.0\end{array}$ \\
\hline
\end{tabular}

lo cual da, en números relativos:

\begin{tabular}{lcccccc}
\hline Lños & Singapur & Formoas & Puerto Rico & Porlodo & \multicolumn{2}{c}{ Koxico } \\
\hline 1953 & 100 & 100 & 100 & $1960-1965$ & 100 & 100 \\
1958 & 92 & 92 & 91 & $1965-1970$ & 86 & 93 \\
1963 & 76 & 80 & 87 & $1970-1975$ & 72 & 85 \\
1968 & 54 & 65 & 71 & $1975-1980$ & 60 & 76 \\
1969 & 50 & 58 & 69 & & & \\
\hline
\end{tabular}

Este cuadro muestra que la hipótesis admitida sobre el descenso de la fecundidad es comparable con la evolución mostrada en realidad en Singapur, Formosa y Puerto Rico.

La proyección demográfica $H 2$ parece, por lo tanto, tener buena posibilidad de realización en el futuro en los países en vías de desarrollo. 
Anexo

TASAS DE FECUNDIDAd POR EDAD UTILIZADAS EN LA HIPÓTESIS $H 2$ (por 1000$)$

\begin{tabular}{|c|c|c|c|c|c|c|c|c|c|c|}
\hline $\begin{array}{c}\text { Grupos } \\
\text { de } \\
\text { edad }\end{array}$ & $\begin{array}{l}1960- \\
1965\end{array}$ & $\begin{array}{r}1965- \\
1970 \\
\end{array}$ & $\begin{array}{r}1970- \\
1975 \\
\end{array}$ & $\begin{array}{r}A \quad \tilde{n} \quad 0 \\
\begin{array}{r}1975- \\
1980\end{array}\end{array}$ & $\begin{array}{r}1980 \\
1985\end{array}$ & $\begin{array}{r}1985- \\
1990\end{array}$ & $\begin{array}{r}1990- \\
1995 \\
\end{array}$ & $\begin{array}{r}1995- \\
2000\end{array}$ & $\begin{array}{r}2000- \\
2005\end{array}$ & $\begin{array}{c}\text { De spué } \\
\text { de } \\
2005\end{array}$ \\
\hline $15-19$ & 105.5 & 96.5 & 87.5 & 79.0 & 70.0 & 61.0 & 52.0 & 43.2 & 34.7 & 34.4 \\
\hline $20-24$ & 304.0 & 288.2 & 273.5 & 258.3 & 243.5 & 228.5 & 214.0 & 199.0 & 189.3 & 187.7 \\
\hline $25-29$ & 315.0 & 289.4 & 264.0 & 238.0 & 212.5 & 187.5 & 161.5 & 135.5 & 112.7 & 111.7 \\
\hline $30-34$ & 273.5 & 245.5 & 217.5 & 191.0 & 162.5 & 134.0 & 106.0 & 77.0 & 55.2 & 54.7 \\
\hline $35-39$ & 198.5 & 176.0 & 153.5 & 131.0 & 109.0 & 86.0 & 64.5 & 42.0 & 25.9 & 25.7 \\
\hline $4 a-44$ & 66.5 & 59.0 & 51.5 & 44.0 & 37.0 & 29.5 & 22.0 & 14.5 & 7.0 & 6.9 \\
\hline $45-49$ & 25.0 & 22.0 & 18.5 & 16.0 & 12.5 & 9.5 & 6.5 & 3.5 & 0.8 & 0.8 \\
\hline Total & 1288.0 & 1176.7 & 1066.0 & 957.8 & 847.0 & 736.0 & 626.5 & 514.7 & 425.5 & 421.9 \\
\hline $\begin{array}{l}\text { T'amaño de la } \\
\text { familia completa }\end{array}$ & 6.4 & 5.9 & 5.3 & 4.8 & 4.2 & 3.7 & 3.1 & 2.6 & 2.1 & 2.1 \\
\hline $\begin{array}{l}\text { Tasa neta de } \\
\text { reproducción }\end{array}$ & 2.60 & 2.43 & 2.28 & 2.12 & 1.92 & 1.70 & 1.46 & 1.21 & 1.01 & 1.00 \\
\hline
\end{tabular}

a Las tablas de mortalidad utilizadas para calcular las tasas netas de reproducción son las mismas que para $H 1$. 